\title{
Effect of increasing fruit and vegetable intake on nitric oxide status: a randomised controlled trial
}

\author{
L. L. Hamill, C. Mc Master, C. E. Neville, A. J. McGrath, C. R. Draffin, A. McGinty, \\ M. C. McKinley and J. V. Woodside \\ Nutrition \& Metabolism Group, Centre for Public Health, Queen's University Belfast BT12 6BJ
}

Epidemiological data indicates that increased intake of fruit and vegetables (FV) is associated with decreased incidence of chronic diseases. This evidence would appear to be strongest for leafy green vegetables for diabetes and cardiovascular disease risk ${ }^{(1-2)}$, and it has been proposed that this may be due to the high nitrate content of such vegetables ${ }^{(3)}$. Increasing evidence supports such a hypothesis and indicates that dietary nitrate, which is subsequently reduced to nitrite and nitric oxide (NO), has the ability to reduce blood pressure and improve endothelial function ${ }^{(4-5)}$. While these studies have focused on beetroot, a particularly rich source of nitrates, very few studies have examined the effect of increased general FV consumption on NO status. The aim of this study was to analyse serum NO in a randomised controlled trial which assessed intake of different portions of FV, to examine the effect of increasing intake on NO status. To do so, we used a subsample from the BIOFAV study.

A total of 20 healthy volunteers were screened, recruited and randomised to consume either $2(n=11)$ or $8(n=9)$ portions of FV/ day for 4 weeks. Fasting serum samples collected at baseline and week 4 were analysed for NO using an ELISA (R and D Systems, Minneapolis, USA) based on the Griess reaction. Between-group and within- group differences were tested using independent and paired sample $t$ tests respectively. Associations between NO at baseline, change in NO and baseline characteristics (age, gender, BMI) were also assessed using Pearson correlation coefficients.

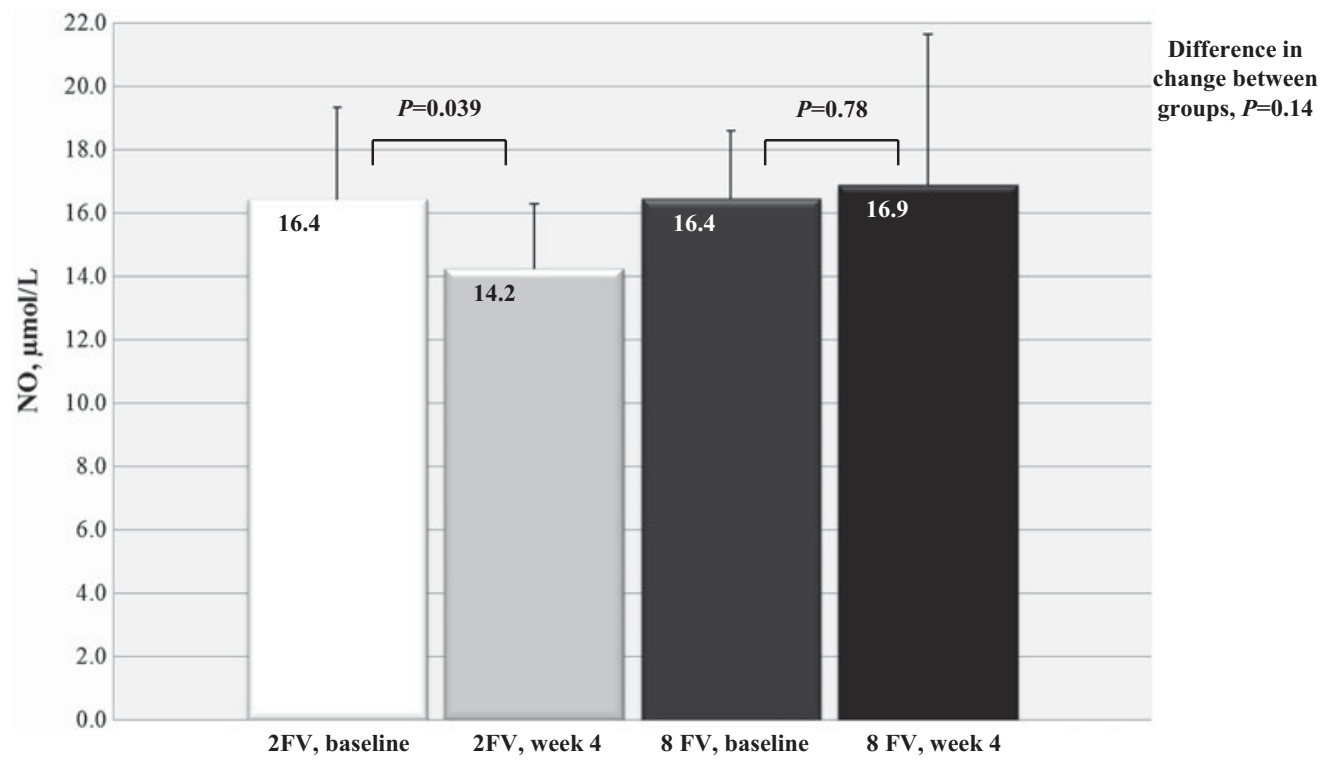

There was no significant difference between groups in change in NO status $(P=0 \cdot 14)$. Those participants who consumed 2 portions FV/day for 4 weeks had a small, but significant $(P=0.039)$ decrease in NO status from baseline while those who consumed 8 portions FV maintained their NO status $(P=0 \cdot 78)$. There were no significant associations between NO at baseline, change in NO and baseline characteristics.

NO might be a useful surrogate marker of systemic NO production after intake of nitrate rich $\mathrm{V}$, but did not respond to an increase in overall FV intake. This study was a small pilot study, and therefore larger numbers of samples may be required to comprehensively establish if increasing FV intake has the potential to alter NO status. Urine NO status may also be of interest, considering that the majority of dietary nitrate is excreted via the kidney.

1. Carter P, Gray LJ, Troughton J et al. (2010) BMJ 341, c4229.

2. Bendinelli B, Masal G, Saieva C et al. (2011) Am J Clin Nutr 93, 275-283.

3. Hord NG, Tang Y \& Bryan NS (2009) Am J Clin Nutr 90, 1-10.

4. Webb AJ, Patel N, Loukogeorgakis S et al. (2008) Hypertension 51, 784-790.

5. Hobbs DA, Kaffa N, George TW et al. (2012) Br J Nutr 108, 2066-2074. 